\title{
Двойная лавинная инжекция в диодных лавинных обострителях
}

\author{
(C) М.С. Иванов, Н.И. Подольская, П.Б. Родин \\ Физико-технический институт им. А.Ф. Иоффре Российской академии наук, \\ 194021 Санкт-Петербург, Россия \\ E-mail: m.ivanov@mail.ioffe.ru
}

Поступила в Редакцию 14 октября 2019 г.

В окончательной редакции 22 октября 2019 г.

Принята к публикации 22 октября 2019 г.

\begin{abstract}
Проведено численное моделирование пикосекундных диодных лавинных обострителей в режиме коммутации быстронарастающих высоковольтных импульсов субмикросекундной длительности. Показано, что максимальная длительность коммутируемого импульса ограничена физическими явлениями, связанными с переходом структуры в режим двойной лавинной инжекции, а не с восстановлением блокирующей способности диода вследствие дрейфового рассасывания неравновесной электронно-дырочной плазмы, как это имеет место в низкочастотных силовых диодах. Двойная лавинная инжекция в принципе способна поддерживать структуру диодного обострителя в проводящем состоянии после переключения. Однако отрицательное дифференциальное сопротивление, имеющее место в этом режиме, влечет за собой неустойчивость однородного токораспределения и изотермическое шнурование тока.
\end{abstract}

Ключевые слова: ударная ионизация, субнаносекундные переключатели, лавинная инжекция, токовые неустойчивости.

DOI: $10.21883 /$ FTP.2020.03.49032.9284

\section{1. Введение}

Явление задержанного лавинного пробоя высоковольтных $p-n$-переходов, открытое в ФТИ им. Иоффе $[1]$, позволило увеличить мощность, коммутируемую полупроводниковыми приборами в субнаносекундном диапазоне, сразу на 5 порядков и привело к созданию новой научно-технической области: мощной импульсной полупроводниковой электроники субнаносекундного диапазона [2-6]. Это явление заключается в быстром (время переключения менее 100 пс) ударно-ионизационном переключении высоковольтной $p^{+}-n-n^{+}$-структуры в проводящее состояние под действием быстро нарастающего импульса обратного напряжения [1,3,7-9]. В момент начала переключения достигается двукратное превышение напряжения на структуре по сравнению с напряжением стационарного лавинного пробоя $U_{a}$. База диодной структуры заполняется неравновесной электронно-дырочной плазмой (ЭДП), концентрация которой многократно превосходит концентрацию легирующей примеси в $n$-базе.

Возникшее в результате быстрой лавинной ионизации проводящее состояние диодного обострителя, вообще говоря, не является самоподдерживающимся, поскольку неравновесная электронно-дырочная плазма находится в обратносмещенной $p^{+}-n-n^{+}$-структуре. Принято считать, что основным процессом, ограничивающим время нахождения диодного обострителя в проводящем состоянии и, следовательно, максимальную длительность импульса тока, который может пропустить структура после переключения, является дрейфовое рассасывание ЭДП [2,3]. Это мнение основано на аналогии с детально исследованным процессом восстановления блокирующей способности низкочастотных силовых диодов при протекании обратного тока [10-12]. В настоящей работе показано, что данная аналогия неверна, и при коммутации диодным обострителем высоковольтных импульсов субмикросекундной длительности восстановления блокирующей способности диода вследствие дрейфового рассасывания не происходит. Главную роль после переключения играют двойная лавинная инжекция и изотермическое шнурование тока.

\section{2. Модель}

Динамика электронно-дырочной плазмы и электрического поля в кремниевом диодном обострителе исследовалась с помощью численного моделирования в рамках диффузионно-дрейфовой модели путем численного решения уравнений непрерывности для электронов и дырок, уравнения Пуассона и уравнений Кирхгофа для внешней цепи. Моделирование проводилось с помощью программного пакета TCAD SILVACO [13]. Схема включения диодного обострителя с последовательной нагрузкой 50 Ом и профиль легирования представлены на рис. 1. Параметры исследуемой структуры отвечают диодным обострителям, исследованным эксперименталь-

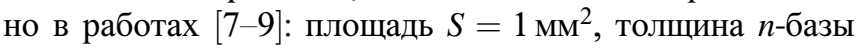
$d=100$ мкм и концентрация легирующей примеси в $n$-базе $N_{d}=1.7 \cdot 10^{14} \mathrm{~cm}^{-3}$. Напряжение стационарного лавинного пробоя такой диодной структуры составляет $U_{a} \approx 1 \kappa \mathrm{B}$.

Приложенный к структуре импульс напряжения $V(t)$ имел квазипрямоугольный вид (рис. 2) и состоял из переднего фронта с наносекундным временем нарастания, за которым следует плато амплитудой $V_{\text {plato }}$. Импульс $V(t)$ был составлен из колоколообразного 


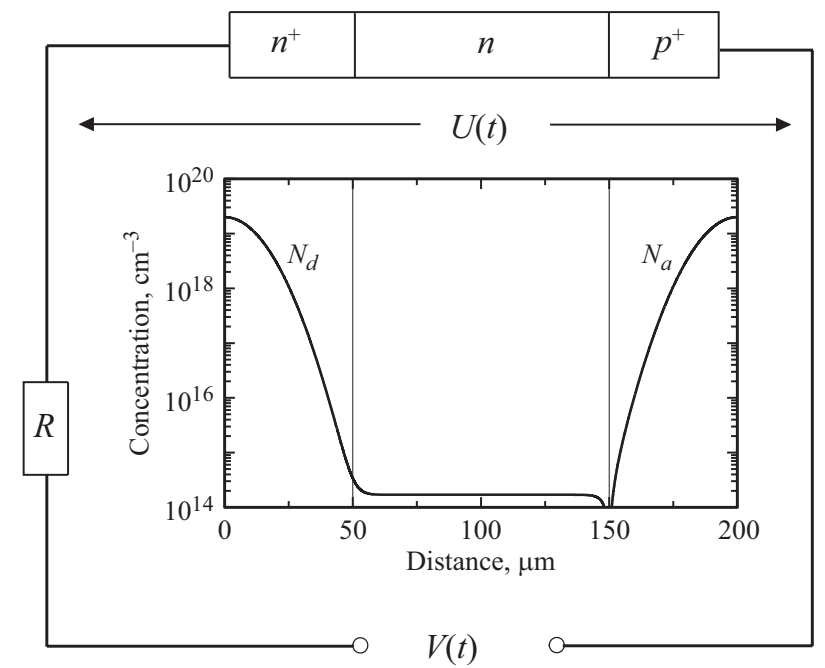

Рис. 1. Схема включения и профиль легирования кремниевой $p^{+}-n-n^{+}$-структуры диодного обострителя импульсов. $V(t)-$ приложенный к структуре и нагрузке $R=50$ Ом импульс напряжения.

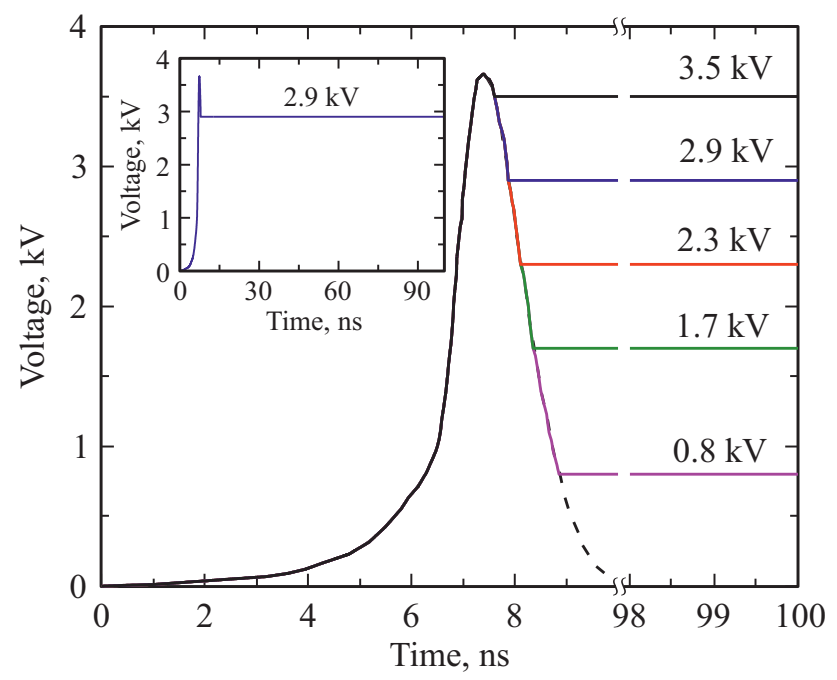

Рис. 2. Импульсы напряжения $V(t)$, приложенные к диоду и последовательной нагрузке $R=50$ Ом. Импульсы совпадают на начальной стадии и отличаются амплитудой $V_{\text {plato }}=800 \ldots 3500$ В на горизонтальном участке. На вставке показан субмикросекундный импульс для напряжения $V_{\text {plato }}=2900 \mathrm{~B}$.

импульса полушириной 1.5 нс и амплитудой 3.7 кВ, применяемого для инициации задержанного ударноионизационного пробоя в экспериментах [7-9], и плато субмикросекундной длительности (рис. 2). Амплитуда плато $V_{\text {plato }}$ изменялась в интервале от 800 В до 3.5 кВ.

\section{3. Результаты одномерного изотермического моделирования}

На рис. 3 показаны напряжение на приборе $U(t)$ и ток $I(t)$ для импульсов $V(t)$ с различной ампли- тудой $V_{\text {plato, }}$ полученные в результате одномерного изотермического численного моделирования при комнатной температуре $T=300 \mathrm{~K}$. Задержанный ударноионизационный пробой начинается в момент $t=6.9 \mathrm{нс}$, когда напряжение на диоде достигает 2.2 кВ, т.е. вдвое превосходит напряжение стационарного пробоя $U_{a} \approx 1$ кВ. За время $\sim 100$ пс напряжение на диоде уменьшается от 2.2 кВ до $500 \mathrm{~B}$, а ток в нагрузке увеличивается до $60 \mathrm{~A}$. Расчетное время лавинного переключения и остаточное напряжение находятся в разумном согласии с экспериментальными результатами, полученными для колоколообразного импульса $V(t)$ [7-9]. Средняя концентрация ЭДП в $n$-базе после переключения составляет $7 \cdot 10^{15} \mathrm{~cm}^{-3}$.

После переключения ток $I(t)$ уменьшается, а напряжение на диоде $U(t)$ нарастает. Однако спад тока не ведет к восстановлению блокирующей способности диода, а завершается переходом в квазистационарное проводящее состояние с постоянным напряжением (рис. 3). Амплитуда тока в этом проводящем состоянии составляет десятки ампер. Напряжение на диоде близко к 1.5 кВ и слабо зависит от величины $V_{\text {plato }}$ Время перехода в стационарное состояние составляет $\sim 10$ нс и уменьшается при увеличении амплитуды импульса $V_{\text {plato }}$.

Анализ пространственного распределения электрического поля и концентраций носителей внутри структуры показывает, что процесс рассасывания ЭДП начинается еще во время движения фронта ионизации. Процесс рассасывания длится $\sim 10$ нс после завершения сверхбыстрого лавинного переключения и приводит к уменьшению концентрации неравновесных носителей в

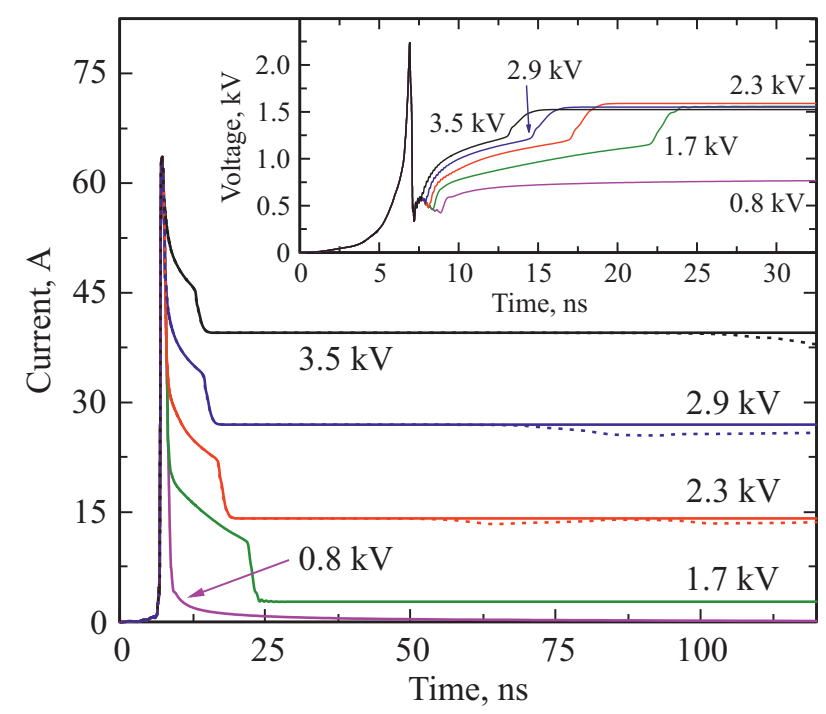

Рис. 3. Зависимости тока $I(t)$ и напряжения $U(t)$ на диодном обострителе от времени при различных амплитудах $V_{\text {plato }}=800 \ldots 3500$ В. Сплошные и штриховые линии отвечают результатам одномерного и двумерного численного моделирования соответственно. Расхождение штриховой и сплошной линий отвечает развитию спонтанной неустойчивости однородного токораспределения и формированию токового шнура, происходящему в двумерном случае. 


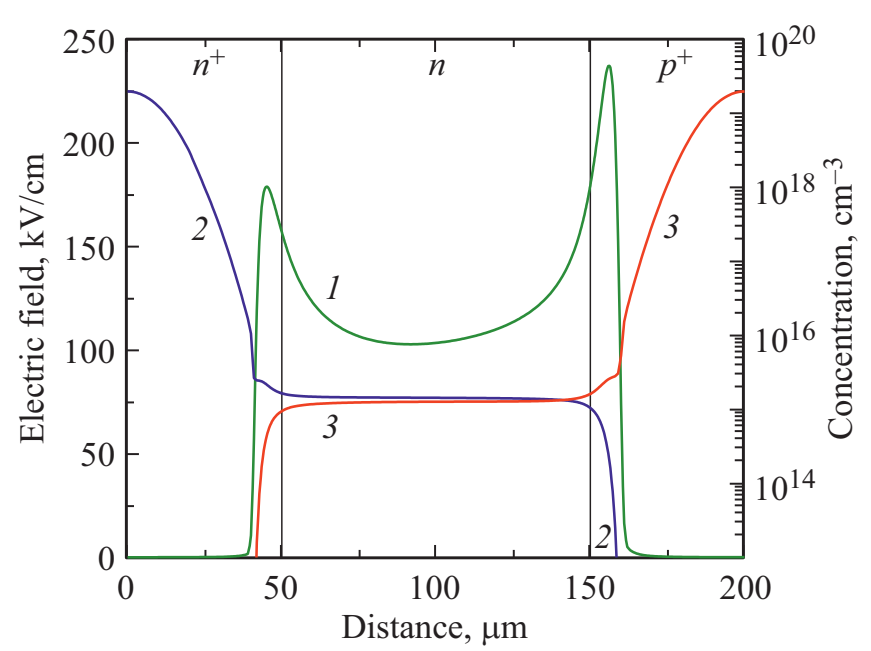

Рис. 4. Распределение электрического поля (зеленая линия 1), концентраций электронов (синяя линия 2) и дырок (красная линия 3) в установившемся квазистационарном состоянии при напряжении $V_{\text {plato }}=3500 \mathrm{~B}$, отвечающем режиму двойной лавинной инжекции. (Цветная версия рисунка - в электронной версии статьи.)

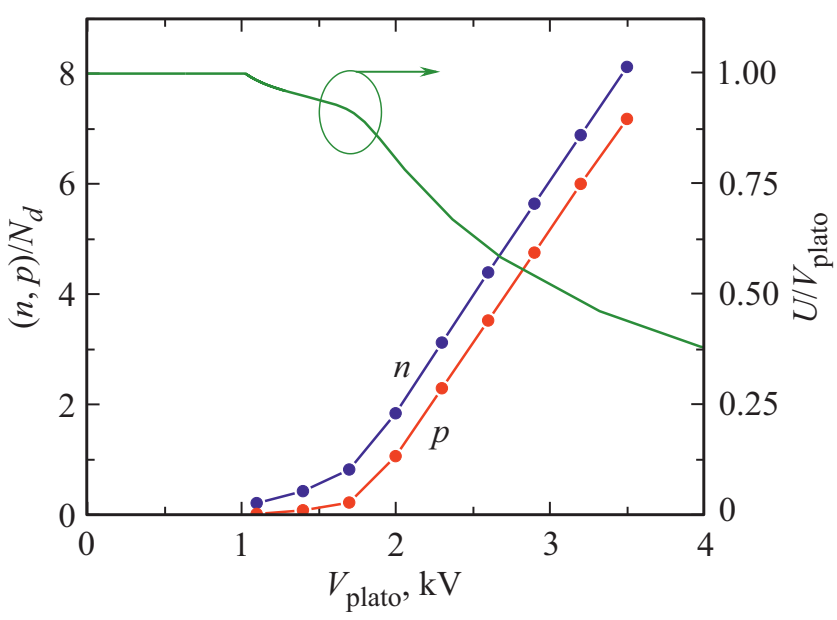

Рис. 5. Зависимость средних по $n$-базе концентраций электронов $(n)$ и дырок $(p)$ и отношения напряжения на диоде $U$ в режиме двойной лавинной инжекции $V_{\text {plato }}$ от напряжения $V_{\text {plato }}$.

несколько раз. Классическая теория [10-12] неприменима к этому процессу, так как рассасывание сопровождается ударной ионизацией в области сильного поля около $p-n$-перехода, что приводит к монополярной лавинной инжекции электронов в базу. Дрейфовое рассасывание не приводит к восстановлению блокирующей способности диода, а прекращается в результате качественной перестройки поля зарядом свободных носителей. Эта перестройка, сопровождаемая ступеньками на зависимостях $I(t)$ и $U(t)$ (рис. 3), приводит к формированию характерного „Двугорбого“ профиля электрического поля (рис. 4) и переходу в квазистационарный режим двойной лавинной инжекции [14]. В показанном на рис. 4 стационарном состоянии у $p^{+}$-контакта в базу за счет ударной ионизации инжектируются электроны, а у $n^{+}$-контакта - дырки.

На рис. 5 показаны зависимость средней концентрации неравновесных носителей в базе структуры и отношение $U / V_{\text {plato }}$ напряжения на приборе к приложенному напряжению при разных значениях амплитуды импуль-

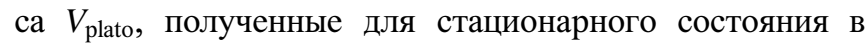
рамках одномерного моделирования. При достаточно больших значениях $V_{\text {plato }}$ концентрация неравновесной плазмы многократно превосходит уровень легирования в базе $N_{d}=1.7 \cdot 10^{14} \mathrm{~cm}^{-3}$, но меньше концентрации $7 \cdot 10^{15} \mathrm{~cm}^{-3}$ неравновесных носителей непосредственно после переключения.

Из рис. 5 следует, что проводимость структуры сильно меняется при увеличении $V_{\text {plato }}$ до величины более $\sim 2$ кВ. Причина ясна из анализа обратной ВАХ диодной структуры, представленной на рис. 6 в логарифмическом масштабе вместе с нагрузочными прямыми. Режим двойной лавинной инжекции отвечает плотности тока более $0.5 \kappa \mathrm{A} / \mathrm{cm}^{2}$ и, при выбранной величине нагрузочного сопротивления 50 Ом, достигается при $V_{\text {plato }} \approx 2$ кВ. Из ВАХ на рис. 6 следует, что при изменении плотности тока в интервале от 0.5 до $10 \kappa \mathrm{A} / \mathrm{cm}^{2}$ (соответствующее изменение полного тока от 5 до $100 \mathrm{~A}$ ) напряжение на диодной структуре меняется в узком интервале значений 1.5-1.6 кВ. Вид обратной BAX в данном интервале токов объясняет слабую зависимость напряжения на обострителе от

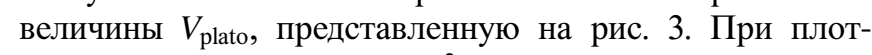
ности тока менее $0.5 \kappa \mathrm{A} / \mathrm{cm}^{2}$ переход в режим двойной лавинной инжекции не происходит, распределение электрического поля в базе имеет единственный максимум вблизи $p-n$-перехода.

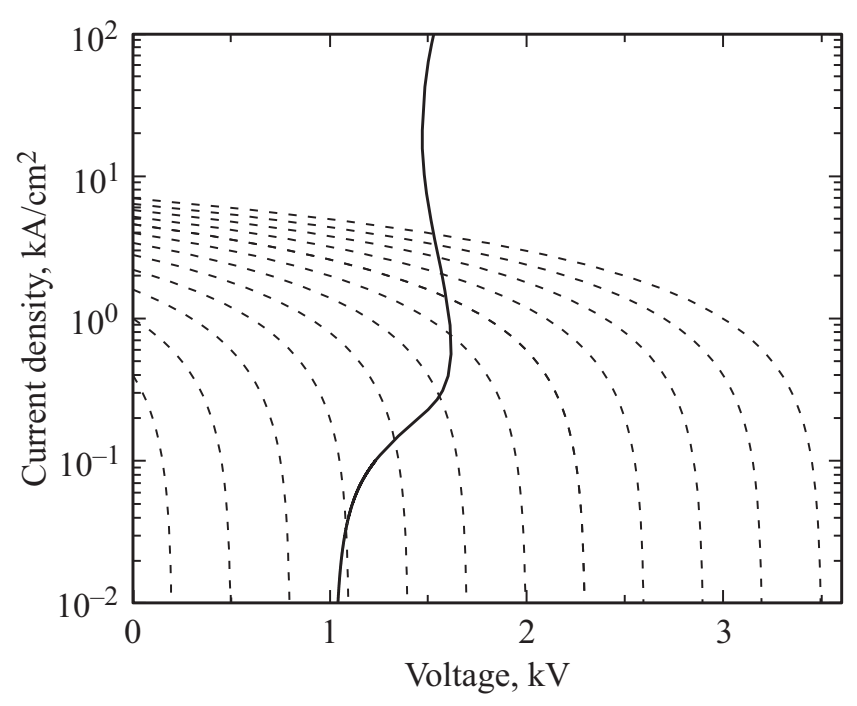

Рис. 6. Обратная изотермическая вольт-амперная характеристика диодного обострителя с параметрами: $S=1 \mathrm{mм}^{2}$, толщина $n$ базы $d=100$ мкм и уровень легирования $N_{d}=1.7 \cdot 10^{14} \mathrm{~cm}^{-3}$. Нагрузочные прямые изображены пунктирными линиями и соответствуют напряжению источника $200-3500$ В с шагом 300 В и нагрузке $R=50$ Ом. 
Как видно из рис. 6, в режиме двойной инжекции обострительный диод имеет отрицательное дифференциальное сопротивление (ОДС). Механизм возникновения ОДС хорошо известен [14] и связан с трансформацией профиля электрического поля при увеличении тока: бо́льшим значениям тока отвечают более высокие и узкие пики электрического поля на краях структуры, обеспечивающие более эффективную лавинную инжекцию, но меньшее значение электрического поля между ними, обусловленное большей концентрацией неравновесных носителей. В результате описанной трансформации напряжение на структуре уменьшается при увеличении тока.

\section{4. Двумерное и неизотермическое моделирование}

Для понимания дальнейшей эволюции состояния прибора одномерное моделирование становится недостаточным, поскольку вследствие ОДС однородное по площади токораспределение неустойчиво, и следует ожидать изотермического шнурования тока. С целью оценить время развития неустойчивости и формирования токового шнура было проведено двумерное численное моделирование. Полученные в результате переходные процессы показаны на рис. 3 штриховыми линиями. Момент начала шнурования отвечает расхождению сплошных линий (одномерное моделирование) и штриховых линий (двумерное моделирование). Видно, что шнурование возникает через несколько десятков наносекунд после переключения и, таким образом, является доминирующим процессом при коммутации субмикросекундного импульса. Шнур имеет характерную ширину несколько десятков микрометров и плотность тока $100 \kappa \mathrm{A} / \mathrm{cm}^{2}$.

При коммутации субмикросекундных импульсов джоулев саморазогрев структуры с хорошей точностью является адиабатическим. Одномерное неизотермическое моделирование, выполненное с учетом джоулева саморазогрева, показывает, что нагрев структуры на этапе до возникновения изотермического шнурования тока при коммутации единичного импульса составляет несколько градусов и, таким образом, не является существенным. Влияние тепла на этапе шнурования тока будет предметом дальнейших исследований. Известно, что в полупроводниковых структурах, отрицательное дифференциальное сопротивление которых связано с ударной ионизацией, стационарный токовый шнур теряет устойчивость относительно сдвига и начинает двигаться по образцу [15-18]. Механизм такого движения может быть связан с уменьшением интенсивности ударной ионизации при росте температуры [15-17]. Возможен также изотермический механизм трансляционной неустойчивости [18]. В обоих случаях миграция шнура по структуре препятствует локальному разогреву и, таким образом, в принципе способна предотвратить или существенно отсрочить тепловую аварию [15-18].

\section{5. Обсуждение результатов}

Основная причина, по которой физические процессы в диодном обострителе импульсов при коммутации ,длинного“ импульса принципиально отличаются от хорошо изученных процессов в низкочастотных силовых диодах при протекании обратного тока, состоит в величине обратного напряжения. Поскольку для переключения диодного обострителя требуется импульс с амплитудой, не менее чем в 2 раза превышающей напряжение стационарного пробоя $U_{a}$, обратное напряжение, приложенное к диоду и последовательной нагрузке после переключения, существенно превосходит $U_{a}$. Именно это в конечном итоге приводит к тому, что ударноионизационные явления - а именно двойная лавинная инжекция - доминируют над дрейфовым рассасыванием после переключения. Восстановление блокирующей способности $p-n$-перехода при столь большом значении обратного напряжения в принципе невозможно. Действительно, оно означало бы прекращение тока через структуру и нагрузку, т.е. увеличение напряжения на диоде до величины, превосходящей $U_{a}$.

\section{6. Заключение}

Проведенное одномерное и двумерное изотермическое численное моделирование приводит к следующим выводам. При коммутации высоковольтных квазипрямоугольных импульсов большой длительности обострительный диод переходит в режим двойной лавинной инжекции, поддерживающей проводящее состояние. Время перехода структуры в режим двойной инжекции составляет около десяти наносекунд. Дифференциальное сопротивление структуры в режиме двойной инжекции отрицательно, что приводит к изотермическому шнурованию тока, которое развивается через несколько десятков наносекунд после переключения. Поэтому фактором, ограничивающим максимальную длительность импульса, коммутируемого диодным обострителем, оказывается не дрейфовое рассасывание плазмы, а резкий рост плотности тока вследствие шнурования.

\section{Благодарности}

Авторы признательны В.И. Брылевскому за многочисленные полезные обсуждения.

\section{Финансирование работы}

Работа поддержана грантом РФФИ № 17-08-01559.

\section{Конфликт интересов}

Авторы заявляют, что у них нет конфликта интересов. 


\title{
Список литературы
}

[1] И.В. Грехов, А.Ф. Кардо-Сысоев. Письма ЖТФ, 5 (15), 950 (1979).

[2] В.М. Тучкевич, И.В. Грехов. Новые принципы коммутации больших мощностей полупроводниковыми приборами (Л., Наука, 1988).

[3] A.F. Kardo-Sysoev. Ultra-Wideband Radar Technology, ed. by J.D. Taylor (London-N. Y.-Washington, CRC Press, Boca Raton, 2001) p. 205.

[4] R.J. Focia, E. Schamiloglu, C.B. Fledderman, F.J. Agee, J. Gaudet. IEEE Trans. Plasma Sci., 25 (2), 138 (1997).

[5] I.V. Grekhov. IEEE Trans. Plasma Sci., 38 (5), 1118 (2010).

[6] А.И. Гусев, С.К. Любутин, С.Н. Рукин, Б.Г. Словиковский, С.Н. Цыранов. ФТП, 48 (8), 1095 (2014).

[7] В.И. Брылевский, И.А. Смирнова, П.Б. Родин, И.В. Грехов. Письма ЖТФ, 40 (8), 80 (2014).

[8] V.I. Brylevskiy, I.A. Smirnova, A.V. Rozhkov, P.N. Brunkov, P.B. Rodin, I.V. Grekhov. IEEE Trans. Plasma Sci., 44 (10), 1941 (2016).

[9] V. Brylevskiy, I. Smirnova, A. Gutkin, P. Brunkov, P. Rodin, I. Grekhov. J. Appl. Phys., 122 (18), 185701 (2017).

[10] H. Benda, E. Spenke. Proc. IEEE, 55, 1331 (1967).

[11] H. Benda, F. Dannhäuser, A. Porst, E. Spenke. Sol. St. Electron., 10 (12), 1133 (1967).

[12] A. Porst, K. Schuster. Sol. St. Electron., 10 (12), 1149 (1967).

[13] SILVACO ATLAS, User guide, http://www.silvaco.com

[14] H.C. Bowers. IEEE Trans. Electron Dev., 15 (6), 343 (1968).

[15] G.K. Wachutka. IEEE Trans. Electron Dev., 38 (6), 1516 (1991).

[16] M. Denison, M. Blaho, P. Rodin, V. Dubec, D. Pogany, D. Silber, E. Gornik, M. Stecher. IEEE Trans. Electron Dev., 51 (8), 1331 (2004).

[17] P. Rodin. Phys. Rev. B, 69 (4), 045307 (2004).

[18] F.J. Niedernostheide, H.J. Schulze. Physica D, 199(1-2), 129 (2004).

Редактор Г.А. Оганесян

\section{Double avalanche injection in diode avalanche sharpeners}

\author{
M.S. Ivanov, N.I. Podolska, P.B. Rodin \\ loffe Institute \\ 194021 St. Petersburg, Russia
}

Abstract Numerical simulations of picosecond-range avalanche sharpening diodes commutating fast-rising high-voltage pulses of submicrosecond duration is performed. It is demonstrated that the maximum duration of the commutated pulse is limited by physical phenomena related to the structure transition to the double avalanche injection mode but not by the drift extraction of nonequilibrium electron-hole plasma. Double avalanche injection is in principle capable of supporting the diode structure in the conducting state after switching. However, negative differential conductivity that is attributed to the double injection mode cause transverse instability of uniform current flow and isothermal current filamentation. 